\title{
ESTUDIO DE LAS TECNOLOGÍAS PARA LA PERSONALIZACIÓN DE MAPAS UTILIZANDO LAS HERRAMIENTAS ACTUALES.
}

\section{STUDY OF TECHNOLOGIES FOR THE PERSONALIZATION OF MAPS USING THE CURRENT TOOLS.}

\author{
Gregorio Patricio Zambrano Álava, Ing. \\ Ingeniero en Sistemas Computacionales (Ecuador).
} Estudiante de Maestría en Sistemas de Información mención Inteligencia de Negocios de la Universidad Tecnológica ECOTEC, Ecuador. gregoriozambrano@yahoo.com

\section{ARTÍCULO DE REFLEXIÓN}

Recibido: 7 de septiembre de 2018.

Aceptado: 28 de noviembre de 2018.

\section{RESUMEN}

Actualmente la tecnología es indispensable en cualquier ámbito científico, debido que ha colaborado con grandes descubrimientos mediante su uso, JavaScript es una de esas tecnologías. La mayoría de las herramientas permiten conocer y analizar puntos georreferénciales expuestos en una cartografía. El objetivo principal de este artículo es identificar cuáles de las herramientas existentes permiten cargar y pintar los puntos georreferenciados en un mapa para la ciudad de Guayaquil utilizando la tecnología de JavaScript. Click2Map, es una herramienta que representa mapas del mundo con la finalidad de dar un análisis permitiendo identificar las zonas o sectores que muestren las calles de la ciudad, una vez que identificadas las herramientas se debe hacer una comparación de la eficiencia o productividad de la misma, la cual permita deducir a traces las ventajas y funciones que ofrece. Click2Maps es una aplicación que representa mapas del mundo proporcionando un análisis en las zonas con más tránsito. Los resultados obtenidos pueden ser usado para dar soluciones a diversos problemas de tránsito vehicular.

Palabras clave: tecnologías, herramientas, JavaScript, Click2Map. 


\section{ABCTRACT}

Nowadays technology is indispensable in any scientific field, because it has collaborated with great discoveries through its use, JavaScript is one of those technologies. Most of tools allow to know and analyze georeferenced points exposed in a cartography. The main objective of this article is to identify which of the existing tools allow to load and paint the georeferenced points on a map for Guayaquil city using JavaScript technology. Click2Map, is a tool that represents Worldwide maps in order to give an analysis allowing to identify the zones or sectors that show the streets of the city, once the tools are identified, a comparison of the efficiency or productivity of them should be made, which can provide the advantages and functions offered. Click2Maps is an application that represents worldwide maps providing an analysis in the areas with most traffic. The results obtained can be used to provide solutions to various vehicular traffic problems.

Keywords: technologies, tools, JavaScript, Click2Map.

\section{INTRODUCCIÓN}

Hoy en día existe una extensa proliferación en cuanto a temas de investigación de diversos disciplinas y áreas del saber, uno de ellos son los sistemas de información que han venido revolucionando este ámbito investigativo de análisis de trayectorias. Estos sistemas de información son orientados al tratamiento y administración de datos e información, organizados y listos para su uso posterior, generados para cubrir una necesidad o un objetivo, el cual será identificar cuáles de las herramientas existentes permiten cargar los puntos georreferenciados en un mapa de la ciudad de Guayaquil utilizando CLICK2Maps. Sin embargo, para llegar a esta herramienta se encontró que existen otras herramientas que permiten utilizar las tecnologías de programación, para explotar y consumir sus recursos se debe trabajar con herramientas que sean compatibles.

Toda organización trabaja de forma constante para ofrecer servicios que den soluciones a los problemas que los aflige, otorgándole una gran importancia a los sistemas de información, que en conjunto trabaja con las diferentes tecnologías que van surgiendo a medida que el tiempo avanza. Ahora bien, la ciudad de Guayaquil presenta índices elevados de tráfico en algunas de las vías de la ciudad, estos índices se han obtenido en base a estadísticas generadas anualmente por las Empresa de Movilidad (EMOV), la Oficina de Investigación de Accidentes de Tránsito (OIAT) y la Comisión de Tránsito del Ecuador (CTE). La Ley Orgánica 
de Transporte Terrestre, Tránsito y Seguridad Vial que proporciona las medidas y políticas para normalizar y proteger a la ciudadanía de los accidentes de tránsito, sin embargo, esto no es suficiente, por ello se propuso hacer un análisis más detallado para tener una medida alterna y controlar esta problemática. Con los datos estadísticos de transporte del 2016 proporcionado por la página del Instituido nacional de estadística y censos (INEC) se pudo analizar que el nivel de tráfico ha ido aumentando en los últimos años. (Véase la figura 1)

\section{Número de vehículos matriculados \\ Periodos: 2008-2016 \\ (Absolutas)}

El número de vehículos matriculados entre los años 2008 y 2016 tuvo un crecimiento anual del $10,6 \%$.

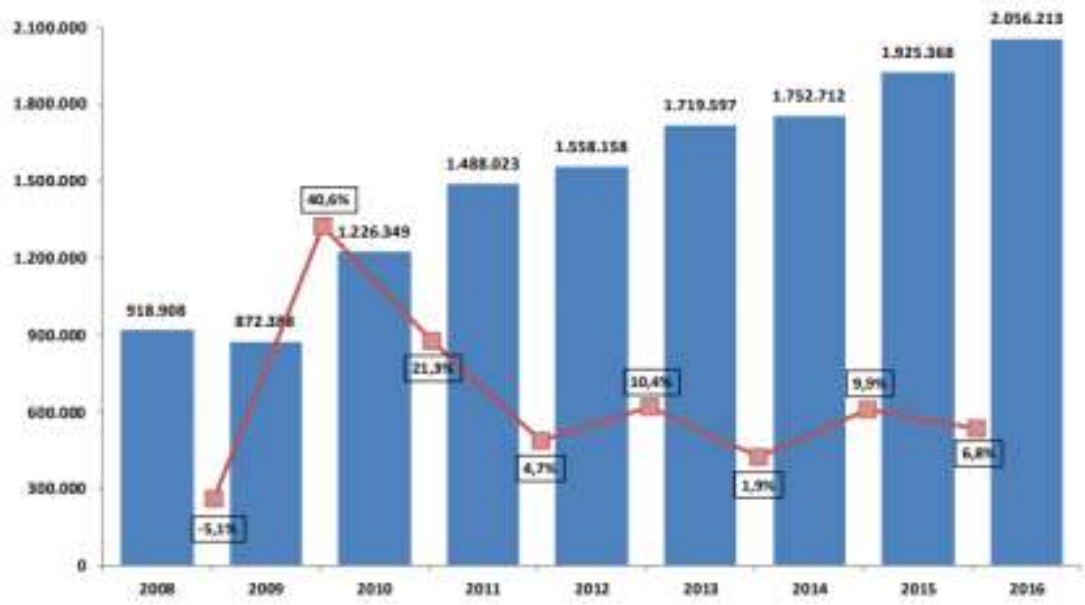

Figura 1. Números de vehículos matriculados. Periodo: 2008-2016.

Fuente: Datos estadísticos INEC (2016).

A partir de la base de esta estadística se definió como objetivo conocer, identificar y seleccionar herramientas factibles para la carga de puntos georreferenciados. Los datos georreferenciados se toman de una lectura de Sistemas de Geoposicionamiento Global (GPS), de mapas u otras fuentes de información. Esta información se obtiene de diversas personas en diversos puntos o por una sola persona, toda esa información puede no tener la calidad deseada para ser procesada por lo que debe regresarse a los colectores originales 0 bien desecharse, lo cual es un desperdicio de recursos.

En otras ocasiones, un solo investigador compila muestras de diversos encuestadores o personal de apoyo y puede tener los mismos problemas, en otras palabras, la información georreferenciada no ha pasado por un control de calidad que verifique que los puntos georreferenciados son los correctos. Este control de calidad es, esencialmente, graficar los 
datos en un mapa de manera que se verifique que las coordenadas geográficas están correctas.

Las herramientas permitirán ofrecer un sin número de ventajas que facilitara dar respuestas a problemas o necesidades que tenga la humanidad. Uno de esos problemas es la fuerte congestión vehicular que existe en las zonas de la ciudad de Guayaquil motivo por el cual se planteó a través de esta investigación determinar una solución o plataforma tecnológica que permita visualizar las zonas con más tráfico; esto se logrará haciendo uso de las herramientas que actualmente forman parte de este ámbito.

Dentro de los resultados alcanzados se presenta a la tecnología JavaScript como opción preferente para el desarrollo de dicha plataforma web debido que es un lenguaje de programación que permite añadir características interactivas a un Sitio Web. Además, se determinó que la herramienta que se usará para este estudio será Click2Map ya que permiten crear mapas profesionales en poco tiempo.

La presente investigación científica pretende la realización de un análisis profundo y crítico en cuanto al uso de las tecnologías para la recolección de datos de los puntos georreferenciados de la ciudad de Guayaquil. Para ello, se realizó revisión documental analizando diversos artículos científicos, textos y libros con la finalidad de determinar cuál tecnología se aplica a los fines de la investigación. Encontrándose que PHP y JAVASCRIPTS son las dos tecnologías que más se adapta al objetivo planteado.

En vista de que ambas tecnologías cuentan con casi las mismas características con respecto a los índices de tiempo que demora en cargar los puntos, y del mismo modo la visualización de ellos para los usuarios. Se procede a evaluar ambas tecnologías con el fin de reconocer cuál de ellas es la mejor y factible para trabajar con los puntos georreferenciados aplicados para trabajar en Click2Maps. Teniendo en cuenta que la carga de los puntos debe ser en el menor tiempo posible, es decir que pueda soportar en cantidad de puntos en corto tiempo sin que caiga la aplicación.

\section{REVISIÓN TEÓRICA}

\subsection{Estudio de Herramientas.}

En esta sección se detalla sobre las herramientas actuales que permiten trabajar con mapas las cuales son: 


\section{Click2Map \\ 2. Map Marker \\ 3. MapPad Pro}

\section{CLICK2MAP}

La interfaz que ofrece Click2Map es completamente sencilla el cual ayuda a crear mapas fácilmente; no se requieren habilidades de programación. Las numerosas funciones de Click2Map le ayudan a crear mapas profesionales en muy poco tiempo. Crea y publica tus mapas en línea, para agregar puntos de interés a cualquier área geográfica de su elección y beneficiarse del poder de una aplicación profesional: importar datos, geo codificar direcciones múltiples realizar actualizaciones por lotes, mostrar direcciones, personalizar su propia base de datos.

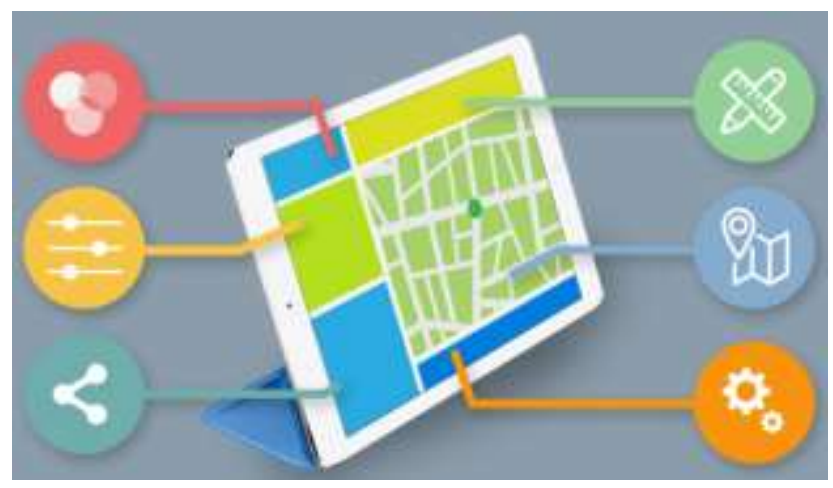

Figura 2. Presentación de la herramienta Click2Map.

Fuente: Click2Map (2006-2018).

Entre ellos se encuentran las siguientes funciones:

\subsection{Personalice sus puntos de interés utilizando las bibliotecas de iconos integrados.}

Personalice sus mapas con iconos de más de 15 bibliotecas de íconos que cubren los temas más comunes (administración, cultura, tiendas, restaurantes, hoteles, turismo, naturaleza, etc.). Los conjuntos de iconos te ayudan a diseñar mapas con contenido claramente organizado y fácilmente reconocible: más de 200 iconos profesionales se proporcionan de manera predeterminada para ayudar a que cada marcador se destaque. 
Los conjuntos de iconos disponibles están organizados en las siguientes categorías:

- Administración, Oficina e Industria

- Cultura y entretenimiento

- Educación y Niños

- Eventos y clima

- Amigos, Familia y Bienes Raíces

- Medios de comunicación

- Misceláneo

- Restaurantes y Hoteles

- Deportes, salud y belleza

- Víveres

- Transporte, etc.

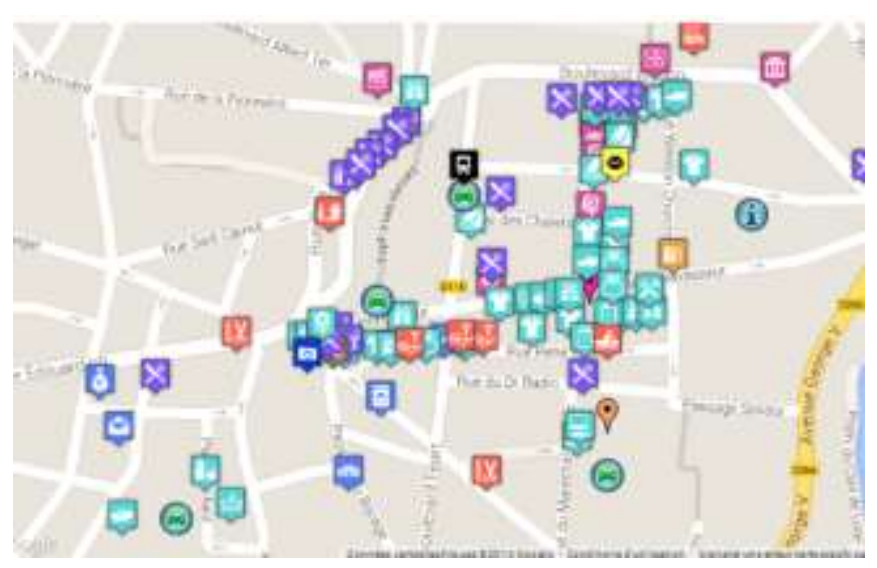

Figura 3. Función personalizar puntos de interés.

Fuente: Click2Map (2006-2018).

\subsection{Mostrar marcadores que contienen texto, fotos, videos.}

Un marcador se puede usar para ubicar un punto de interés o una posición, que se representa gráficamente mediante un ícono en su mapa. Un marcador puede contener diferentes tipos de información, como textos, imágenes, código HTML.

- Fotos, videos y texto en la descripción

- Agrega pestañas personalizadas con foto, video, código HTML 
- Mostrar u Ocultar dirección, coordenadas

- Cambiar propiedades adicionales: correo electrónico, sitio web, etiquetas.
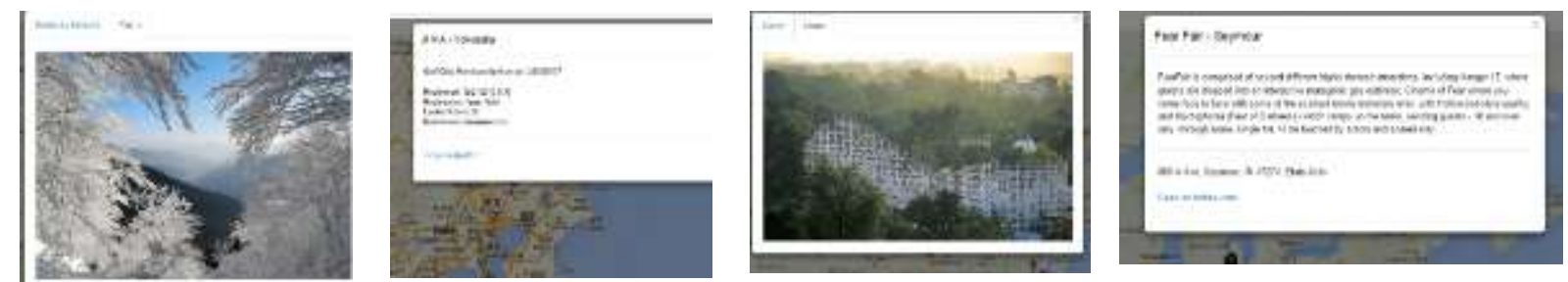

Figura 4. Presentación de la herramienta Click2Map. Función mostrar marcadores Fuente: Click2Map (2006-2018).

\section{Dibujar líneas y polígonos.}

Click2Map Editor le permite dibujar líneas, polilíneas y polígonos en sus mapas. Las herramientas de Dibujo se pueden utilizar para diseñar formas simples y complejas, para resaltar las áreas y dibujar pistas e itinerarios directamente en sus mapas. Las opciones de dibujo le permiten:

- Cambiar la opacidad de la línea y el color de relleno de un polígono

- Elige colores

- Cambiar el peso de las líneas

- Agregue una descripción con texto, fotos o videos: al igual que un marcador, puede describir su dibujo y poner una foto o un video para ilustrarlo.

- Mostrar u ocultar líneas y polígonos

- Al hacer clic en un dibujo, se abre su ventana de información y se muestra la información asociada a él.
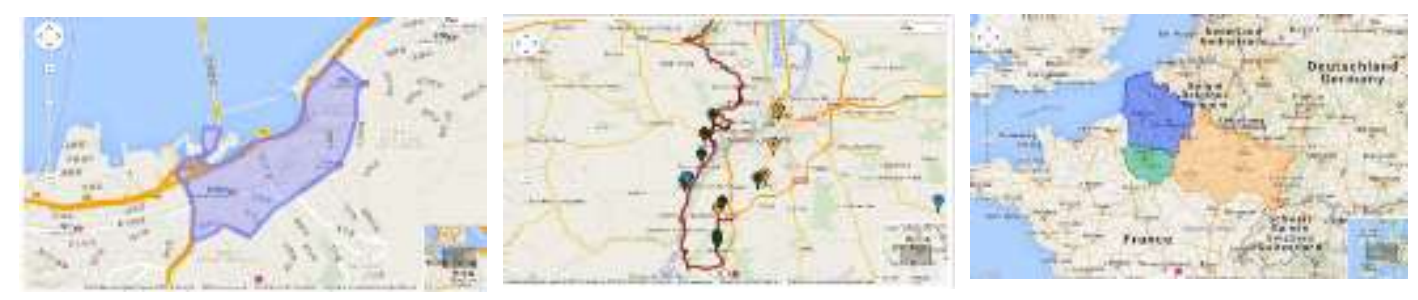

Figura 5. Función dibujar línea y polígonos.

Fuente: Click2Map (2006-2018). 


\section{Organice sus puntos de interés en grupos.}

Click2Map le permite organizar sus marcadores en grupos y subgrupos completamente personalizables para hacer que trabajar con un gran número de marcadores sea más eficiente.

Los grupos tienen propiedades que se pueden definir individualmente: diferentes grupos pueden, por ejemplo, usar un icono predeterminado diferente, lo que hace que sea fácil representar visualmente marcadores con propiedades comunes.

Los grupos se pueden mostrar / ocultar fácilmente para que pueda visualizar solo los marcadores que necesita en su mapa. Los grupos también son excelentes para el funcionamiento masivo: seleccionar todos los marcadores dentro de un grupo es muy fácil. Los grupos se pueden crear dentro de grupos para una organización aún más precisa de sus marcadores.

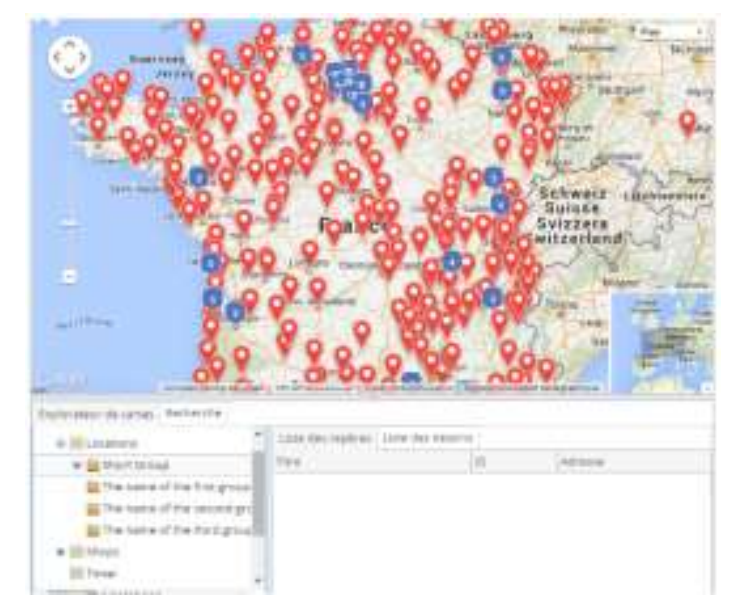

Figura 6. Función organizar puntos en grupos.

Fuente: Click2Map (2006-2018).

\section{Crea puntos de interés directamente desde sus coordenadas geográficas.}

Click2map le permite colocar marcadores con precisión en coordenadas geográficas dadas, si los conoce, se pueden usar coordenadas decimales y grados.

La forma más precisa de posicionar un marcador o punto de interés es especificar sus coordenadas geográficas: para hacerlo, abra las propiedades de un marcador y use los campos de entrada de Latitud y Longitud para especificar sus coordenadas. 


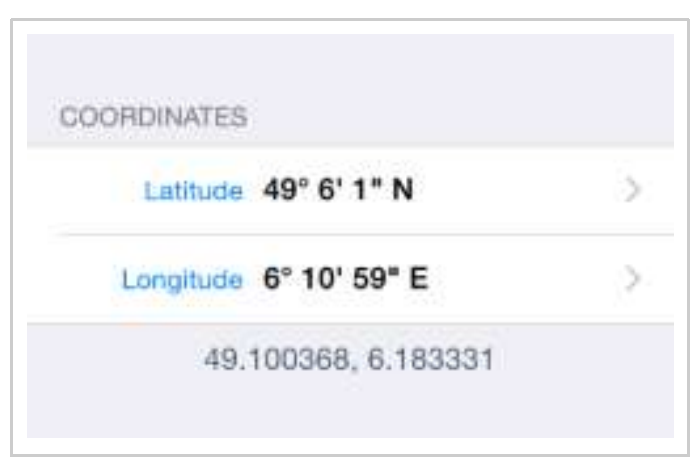

Figura 7. Función crear puntos desde sus coordenadas.

Fuente: Click2Map (2006-2018).

4 Usa el módulo GPS de tu dispositivo para centrar el mapa en tu ubicación actual.

Click2Map Mobile se puede configurar para usar el módulo GPS de su dispositivo para centrar el mapa en su ubicación geográfica; presionar un botón es todo lo que necesita hacer para agregar un PDI en su ubicación exacta.

Aproveche el módulo GPS de su dispositivo para crear marcadores fácilmente en los lugares que visita. El módulo GPS de su dispositivo también es de gran ayuda cuando está en movimiento y solo desea centrar el mapa para identificar los PDI creados previamente en su ubicación.

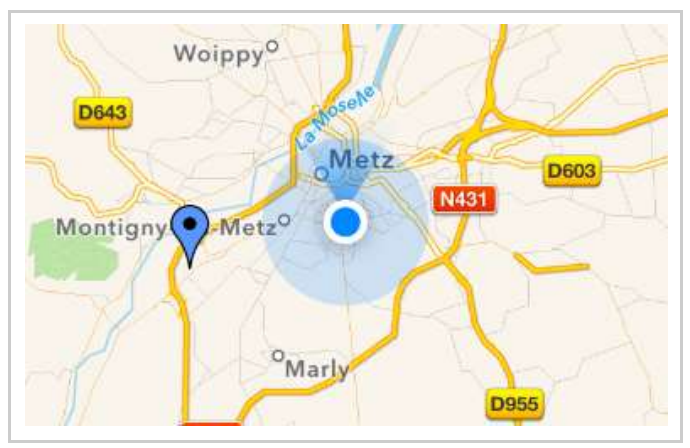

Figura 8. Función modulo GPS.

Fuente: Click2Map (2006-2018).

Extensas estadísticas en sus mapas y marcadores.

Los mapas alojados por Click2Map ofrecen estadísticas completas de tráfico y uso hasta el nivel de marcador: número de visitas, visitantes únicos, número de clics, tiempo invertido, etc. Puede medir fácilmente el impacto de su mapa en sus clientes, socios, etc. 


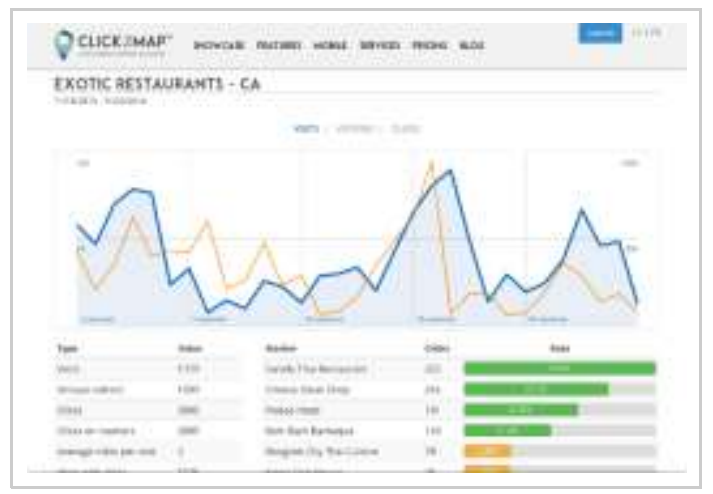

Figura 9. Función estadística en mapas.

Fuente: Click2Map (2006-2018).

Importar bases de datos de direcciones en una gran selección de formatos.

El potente módulo de importación de Click2Map le permite importar cientos e incluso miles de marcadores en un mapa en unos sencillos pasos. Se pueden usar múltiples formatos de datos para importar y actualizar información de marcador: CSV (texto / Excel), KML, GeoRSS y XML.

El módulo de importación detecta automáticamente los campos de datos más comunes en función de su encabezado: una vez configurado correctamente, su archivo de datos se puede importar para crear nuevos marcadores o actualizar los existentes sin problemas.

Click2Map maneja una cantidad de campos de datos predeterminados, pero puede agregarlo si lo necesita: el módulo de base de datos personal puede ampliarse para manejar sus propios datos y mejorar las capacidades del módulo de importación.
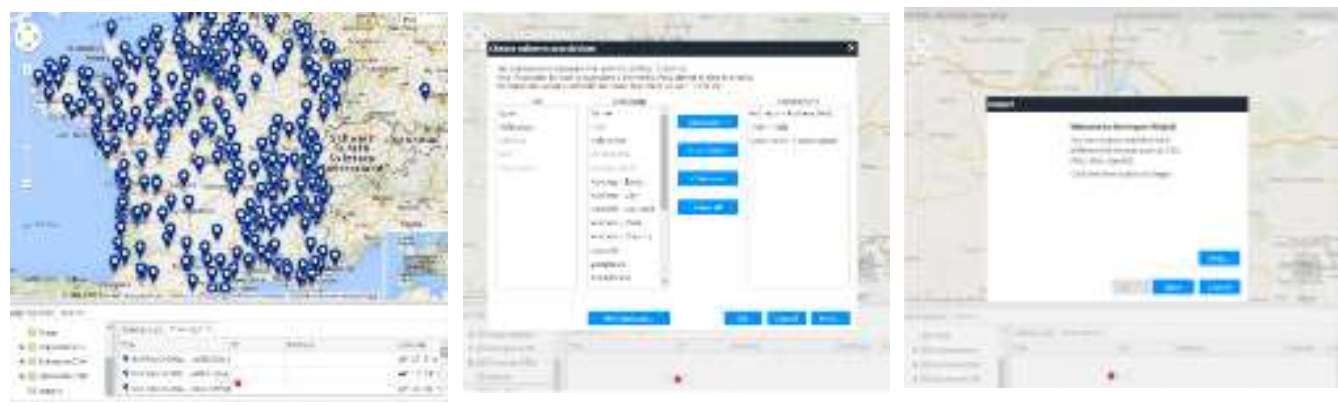

Figura 10. Función importar datos.

Fuente: Click2Map (2006-2018). 
Cabe recalcar que, la geocodificación de una dirección convierte una dirección de calle en coordenadas geográficas (latitud / longitud) que luego pueden usarse para posicionar un marcador en un mapa. Un marcador puede ubicarse en un mapa ingresando sus coordenadas geográficas o geocodificando una dirección de calle. También es posible posicionar manualmente un marcador sin usar geocodificaciones.

\subsection{SERVICIOS CLICK2MAP}

Nuestra experiencia basada en el desarrollo de la aplicación Click2Map y sus miles de usuarios es alabada internacionalmente. Nuestra experiencia única garantiza una ejecución rápida y rigurosa de todas las necesidades de desarrollo de su computadora.

\section{Map Marker}

Es una herramienta lanzada por la empresa Google en junio del 2008. Fue diseñada para mejorar el servicio que ofrece Google Maps. Su objetivo era recopilar la mayor cantidad posible de información de calidad suministrada por los propios usuarios, que luego era verificada por moderadores para posteriormente ser publicada en Google Maps. Esta herramienta fue cerrada el año pasado, pero de toda forma fue una herramienta que facilitó el trabajo con mapas. Cabe mencionar que todas las funcionalidades que tenía migraron a Google Maps.

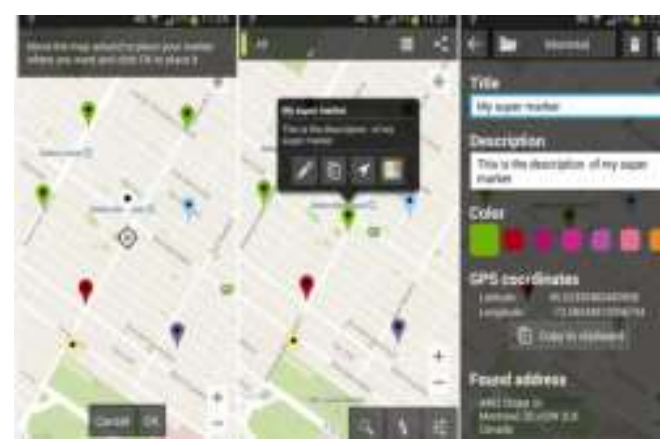

Figura 11. Herramienta Map Marker.

Fuente: Mappinggis (2017).

\section{MapPad Pro}

Esta herramienta crear mapas personalizados en los que te interese dibujar sobre el terreno, o calcular la altitud o distancia de una zona en concreto, dado que usa muchas fuentes como 
mapas base y dispone de varios métodos de medición, aunque vengan acompañados por pagos in-app. El objetivo principal de la aplicación MapPad es la medición, sin embargo, la aplicación se puede utilizar también para muchos otros propósitos relacionados con la planificación de actividades deportivas y al aire libre.

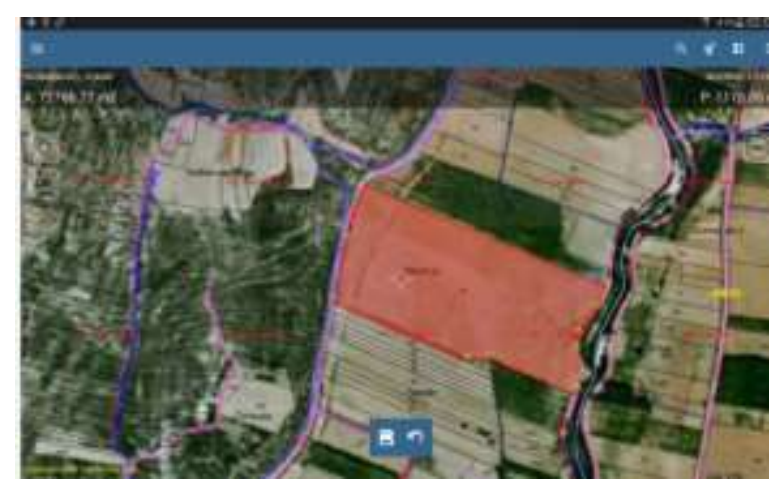

Figura 12. Herramienta MapFad Pro.

Fuente: Mappinggis (2017).

Sus principales características son:

- Mostrar mapa: en este momento, la aplicación puede mostrar 9 mapas de fondo diferentes de 3 proveedores de datos diferentes: Mapa de calles abiertas.

- Mapas sin conexión: la aplicación puede mostrar mosaicos de mapas personalizados preparados en formato OSM Mapnik, los cuales están fuera de línea o mapas sin conexión: método alternativo (No recomendado para niveles de zoom más altos)

- Exportación de datos: MapPad tiene varias opciones de exportación. Las mediciones registradas de longitud o área se pueden exportar utilizando los siguientes formatos KML, GPX, CSV y DXF. También puede compartir los datos directamente utilizando Google Fusion Tables y subir también sus pistas directamente a Click2Maps. Los archivos exportados también se pueden compartir usando G Drive, G Mail o Ever Note, correo electrónico o Skype.

- Entre otras funcionalidades más.

\section{Tecnologías}

Para hacer uso de las herramientas es necesario tener un ambiente de desarrollo compatible, en el cual se pueda reducir código de desarrollo y sobre todo consumir las librerías que facilitan las herramientas mencionadas anteriormente. Se encontró dos tecnologías que 
trabajan con esas herramientas cumpliendo con el objetivo de pintar o dibujar mapas que representen la problemática definida al inicio de este estudio. Entre las tecnologías se encuentran las siguientes:

\section{JavaScript}

Es un robusto lenguaje de programación que puede ser aplicado a un documento $\underline{H T M L}$ y usado para crear interactividad dinámica en los sitios web.

JavaScript por si solo es bastante compacto, aunque muy flexible, y los desarrolladores han escrito gran cantidad de herramientas encima del núcleo del lenguaje JavaScript, desbloqueando una gran cantidad de funcionalidad adicional con un mínimo esfuerzo. Esto incluye:

- Interfaces de Programación de Aplicaciones del Navegador (APIs) - APIs construidas dentro de los navegadores que ofrecen funcionalidades como crear dinámicamente contenido HTML y establecer estilos CSS, hasta capturar y manipular un vídeo desde la cámara web del usuario, o generar gráficos 3D y muestras de sonido.

- APIs de Terceros, que permiten a los desarrolladores incorporar funcionalidades en sus sitios de otros proveedores de contenidos como Twitter o Facebook.

- Marcos de trabajo y librerías de terceros que puedes aplicar a tu HTML para que puedas construir y publicar rápidamente sitios y aplicaciones.

Además, en los últimos años JavaScript se está convirtiendo también en el lenguaje "integrador". Lo encontramos en muchos ámbitos, ya no solo en Internet y la Web, también es nativo en sistemas operativos para ordenadores y dispositivos, del lado del servidor y del cliente. Aquella visión de JavaScript "utilizado para crear pequeños programitas encargados de realizar acciones dentro del ámbito de una página web" se ha quedado muy pequeña.

En el contexto de un sitio web, con JavaScript puedes hacer todo tipo de acciones e interacción. Antes se utilizaba para validar formularios, mostrar cajas de diálogo y poco más. Hoy es el motor de las aplicaciones más conocidas en el ámbito de Internet: Google, Facebook, Twitter, Outlook... absolutamente todas las aplicaciones que disfrutas en tu día a día en la Web tienen su núcleo realizado en toneladas de Javascript. La Web 2.0 se basa en el uso de JavaScript para implementar aplicaciones enriquecidas que son capaces de realizar todo tipo de efectos, interfaces de usuario y comunicación asíncrona con el servidor por medio de Ajax. 


\section{PHP}

PHP es el acrónimo recursivo en inglés de PHP Hypertext Preprocessor (preprocesador de hipertexto), es un lenguaje de programación de propósito general de código del lado del servidor originalmente diseñado para el desarrollo web de contenido dinámico. Fue uno de los primeros lenguajes de programación del lado del servidor que se podían incorporar directamente en un documento HTML en lugar de llamar a un archivo externo que procese los datos. El código es interpretado por un servidor web con un módulo de procesador de PHP que genera el HTML resultante.

\section{Características}

- Orientado al desarrollo de aplicaciones web dinámicas con acceso a información almacenada en una base de datos.

- Es considerado un lenguaje fácil de aprender, ya que en su desarrollo se simplificaron distintas especificaciones, como es el caso de la definición de las variables primitivas, ejemplo que se hace evidente en el uso de php arrays.

- El código fuente escrito en PHP es invisible al navegador web y al cliente, ya que es el servidor el que se encarga de ejecutar el código y enviar su resultado HTML al navegador.

- Capacidad de conexión con la mayoría de los motores de base de datos que se utilizan en la actualidad, destaca su conectividad con MySQL y PostgreSQL.

- Capacidad de expandir su potencial utilizando módulos (llamados ext's o extensiones).

- Posee una amplia documentación en su sitio web oficial, entre la cual se destaca que todas las funciones del sistema están explicadas y ejemplificadas en un único archivo de ayuda.

- Es libre, por lo que se presenta como una alternativa de fácil acceso para todos.

- Permite aplicar técnicas de programación orientada a objetos.

- No requiere definición de tipos de variables, aunque sus variables se pueden evaluar también por el tipo que estén manejando en tiempo de ejecución.

- Tiene manejo de excepciones (desde PHP5).

- Si bien PHP no obliga a quien lo usa a seguir una determinada metodología a la hora de programar, aun haciéndolo, el programador38 puede aplicar en su trabajo cualquier técnica de programación o de desarrollo que le permita escribir código ordenado, estructurado y manejable. Un ejemplo de esto son los desarrollos que en PHP se han 
hecho del patrón de diseño Modelo Vista Controlador (MVC), que permiten separar el tratamiento y acceso a los datos, la lógica de control y la interfaz de usuario en tres componentes independientes.

- Debido a su flexibilidad ha tenido una gran acogida como lenguaje base para las aplicaciones WEB de manejo de contenido, y es su uso principal.

\section{Similitud entre ambas tecnologías}

- Interpretado. Tanto PHP como JavaScript son lo que usted llama idiomas interpretados, o "scripts". el código se puede ejecutar como está en sus respectivos entornos de tiempo de ejecución (navegador para JavaScript, servidor para PHP). Si bien hay muchos matices en el debate compilado vs. el debate interpretado, generalmente es cierto que los guiones son mucho más fáciles de usar y favorecen la productividad del programador. Tanto PHP como JavaScript entran en esta categoría y son excelentes para principiantes y veteranos por igual.

- Ubicuo. Juntos, tanto PHP como JavaScript accionan la mayoría de los sitios web. Durante la mayor parte de su existencia, fueron como mantequilla de maní y jaleaJavaScript en el front-end, PHP en el back-end. Eso significa que hay una gran base de código de bibliotecas, marcos y compatibilidad con la comunidad para estos dos idiomas como un paquete. Piense en una idea o pregunta y es probable que alguien, en alguna parte, haya desarrollado una solución.

\section{Principales Diferencias}

- Entornos de tiempo de ejecución. Hay una razón por la que JavaScript es casi sinónimo de scripting del lado del cliente, mientras que PHP se ha mantenido en gran medida en el lado del servidor. Si bien ambos se pueden incrustar directamente en HTML, ambos necesitan un intérprete para poder ejecutar. El intérprete de JavaScript está integrado en todos los principales navegadores web porque es un componente central de la experiencia web. Entornos de tiempo de ejecución. Si bien JavaScript y PHP se pueden incrustar directamente en HTML, ambos necesitan un intérprete para poder ejecutar. Durante mucho tiempo, PHP ha sido fácil de instalar y usar en el lado del servidor, y está impulsado por el motor Zend.Node.js fue un elemento que cambió 
el juego porque permitía que JavaScript se ejecutara en el lado del servidor, pero antes de 2009, su uso se consideraba en gran parte restringido al desarrollo de front-end.

- Sencillez. PHP es conceptualmente mucho más simple de usar que Node.js. Cuando configura un servidor, todo lo que necesita es un archivo ".php" con un código envuelto entre las <?php ?> etiquetas, ingrese la URL en su navegador, y listo. La declaración que envuelve entre esas etiquetas puede ser tan simple como <?php echo 'Hello World'; ?>y funcionará. Detrás de escena, un servidor web como MySQL con PHP instalado podrá interpretar el archivo y mostrar su página web en su navegador. La configuración de un servidor Node.js, aunque no es difícil, generalmente requiere más líneas de código y una comprensión básica de cómo funcionan las funciones de cierre y devolución de llamada.

- Concurrencia PHP, como la mayoría de los lenguajes del lado del servidor, usa E / S de bloqueo de múltiples hilos para llevar a cabo múltiples tareas en paralelo. JavaScript es único en el sentido de que utiliza algunos trucos (bucle de eventos + agrupamiento de nodos) para lograr un modelo de ejecución de E / S no bloqueado y controlado por eventos que utiliza un solo hilo principal de ejecución. PHP ha estado alrededor del bloque por un tiempo, y ha encontrado su propia forma de lograr un procesamiento asíncrono, especialmente a través del proyecto HHVM lanzado por Facebook.

- JSON. JSON "JavaScript Object Notation" es un formato de datos liviano que es sintácticamente similar a las definiciones de objetos JavaScript. Naturalmente, eso le da una ventaja a las tecnologías basadas en Node.js y JavaScript cuando se trata de JSON. Si bien PHP puede funcionar con JSON, es más situacional.

Ahora bien, una vez analizada las características de las dos tecnologías presentadas se debe saber cuáles de ambas será factible para el desarrollo de la creación de mapas según la herramienta seleccionada. En ese sentido, se establece las siguientes aclaraciones:

5 JavaScript para el lado del cliente y PHP para el lado del servidor. Pero, si está tratando de elegir entre tecnologías de back-end o está construyendo una pila de soluciones completa, ayuda entrar en detalles un poco más.

6 Considerar PHP si el proyecto involucra los CMS (Content Management System Sistema de Gestión de Contenido) como WordPress, Drupal o Joomla, etc.

7 Entre ellas están:

- Servidores como MySQL, SQL, MariaDB, Oracle, Sybase y Postgresql, etc.

- Pilas de soluciones como LAMP (Linux, Apache, MySQL, PHP) 
- Debes considerar JavaScript si el proyecto involucra aplicaciones dinámicas de una sola página (SPA) tales como:

- Tecnologías de front-end como jQuery, AngularJS, Backbone.js, Ember.js, ReactJS, etc.

- Tecnologías del lado del servidor como Node.js, MongoDB, Express.js, etc.

- Pilas de soluciones como MEAN (MongoDB, Express.js, AngularJS)

Con toda la investigación base que se ha ido recolectando se establece los elementos de comparaciones entre las dos tecnologías que permitan desarrollar y verificar cuál de ellas es factible de usar y así cumplir con el objetivo de cargar una gran cantidad de puntos en el menor tiempo posible, sin que el programa se pause o se dañe. Para ello se hace un análisis previo de los lenguajes compatibles con Click2Maps y todo su entorno, luego se procede a obtener los datos necesarios para poder hacer la comparación.

Para un mejor análisis se necesitará de gráficos que permitan visualizar los resultados de una forma más clara. El siguiente gráfico presenta los resultados obtenidos en base a una investigación previa del tiempo de carga de puntos en ambas tecnologías. Como lo muestra la siguiente ilustración:

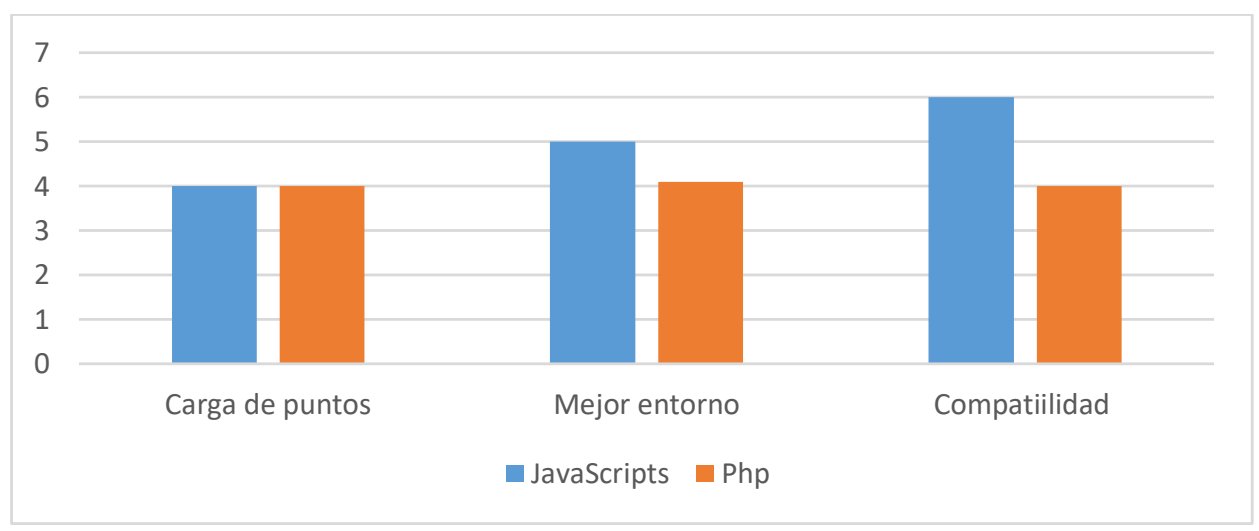

Figura 13. Comparación de puntos PHP y JAVASCRIPT

Fuente: Elaboración propia.

Una vez que comparamos la carga de puntos entre ambos lenguajes de desarrollo, y que descubrimos cuál de las dos tecnologías es más eficiente para la carga de puntos y demás características, se puede ahora representar la productividad que tendrá si usamos JAVASCRIPT vs PHP en nuestro mapa de la aplicación CLICK2Maps. 


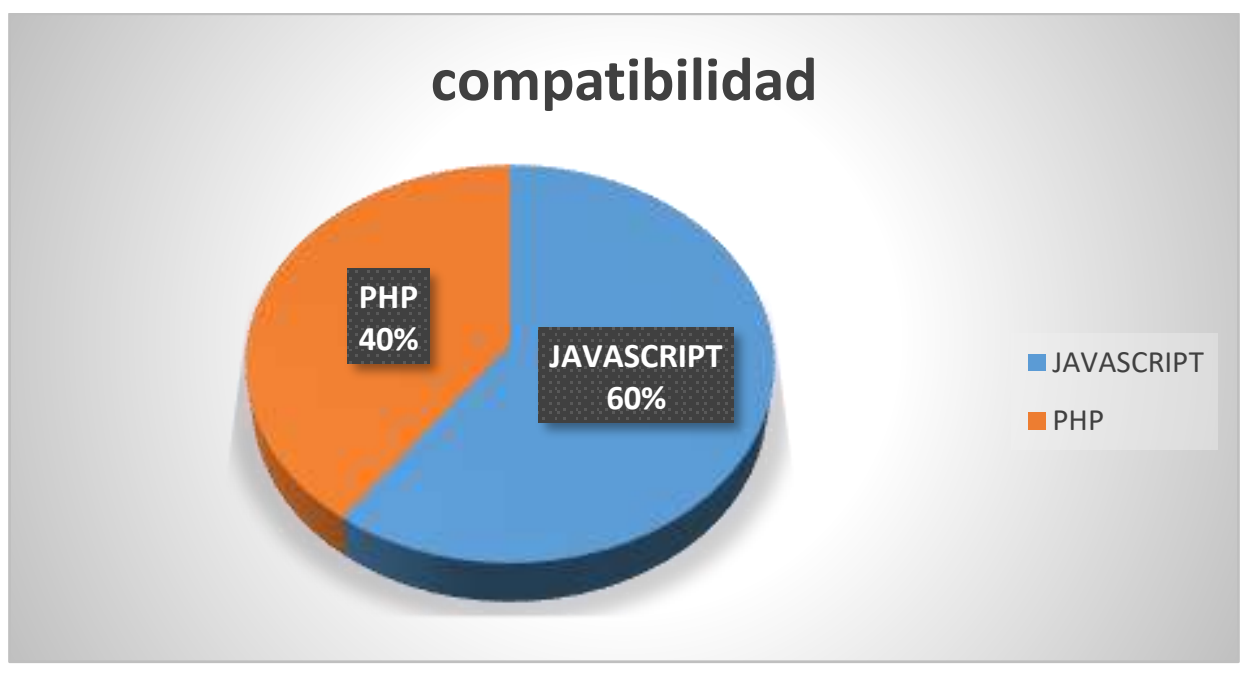

Figura 14. Resultados de la productividad de puntos PHP y JAVASCRIPT Fuente: Elaboración propia.

Después de lograr hacer la comparación entre PHP y JAVASCRIPTS y conocer cuál es el resultado de la misma, podemos descubrir cómo esta investigación tiene relación con la inteligencia de negocios, en donde se conoce como concepto básico que es un conjunto de procesos, aplicaciones y tecnologías que facilitan la obtención rápida y sencilla de datos provenientes de los sistemas de gestión empresarial para su análisis e interpretación, de manera que puedan ser aprovechados para la toma de decisiones y se conviertan en conocimiento para los responsables del negocio. (Castro, 2015)

Esta tecnología actúa como un factor clave y estratégico para la organización ya que provee a los tomadores de decisiones de información oportuna y confiable para responder a las situaciones que puedan presentarse en la empresa como son la entrada a nuevos mercados, el análisis de costos, la rentabilidad de una línea de productos, etc. (Castro, 2015)

A partir de la relación presentada se considera que puede desarrollarse una aplicación que permita mitigar la problemática de congestión vehicular, dado que aún no se soluciona. Se recomienda presentar el análisis realizado a las entidades de transporte competente públicas o privadas, generando conocimiento sobre las posibles soluciones de tránsito vehicular de la ciudad de Guayaquil.

Así mismo, se permite conocer un poco más sobre el uso adecuado de algunas de las herramientas que facilita la inteligencia de negocios para este tipo de investigaciones en el que se pueda marcar una gran diferencia entre una empresa que logra el crecimiento y una 
que no lo hace, en este caso logrará que las empresas de transporte puedan controlar vías colapsadas y ofrecer rutas alternas o un análisis para resolver por ahora la problemática de congestión vehicular a través del uso de herramientas tecnologías, de igual forma a un futuro se podría levantar un análisis que genere conocimiento sobre otras soluciones de transito tales como la construcción de puentes alternos, señalización, semaforización, definición de las vías, de ser necesario poderlas cambiar a una sola vía o doble vía según sea la necesidad, tenido como resultado un mejor control de tráfico de las vías de la ciudad de Guayaquil y a la ciudadanía en contento sin dejar a un lado el renombre, éxito y buena gestión de la entidad de tránsito.

\section{CONCLUSIONES}

La investigación realizada deja fuertes cimientos con resultados comprobados, en donde el uso de las tecnologías facilita las comunicaciones y el intercambio de información, además de que automatiza y acelera los procesos de calidad siendo más exhaustivos en su análisis. Por otra parte, también se logró identificar cuál de las tecnologías expuestas cumple con lo necesario para cargar los puntos georreferenciado en el menor tiempo posible, sin que la aplicación CLICK2MAPS se pause o se cuelgue.

Entonces se deduce que JAVASCRIPT es la mejor opción para la carga de puntos georreferénciales gracias a su fácil entorno de desarrollo y gran compatibilidad con la herramienta de creación de mapas, además se sabemos que tiene múltiples funcionalidades.

Las investigaciones realizadas y los resultados obtenidos puedan ser utilizados para otros investigadores que tengan o se dirijan hacia el tránsito, en donde se hizo un análisis profundo desde su concepción hasta llegar al objetivo que se planteó en el principio de este ensayo, del modo que se creé nuevos conocimientos de las herramientas que actualmente existen para representar puntos en un mapa y que este genere un análisis de acuerdo a las especificaciones o resultados que se desee tener para la solución de alguna otra problemática.

\section{REFERENCIAS BIBLIOGRÁFICAS}

Anónimo. (Junio de 2001). PHP. Obtenido de http://php.net/manual/es/intro-whatis.php Anónimo. (2018). MappApp. Obtenido de http://mappadapp.com/ 
Castro, J. (12 de Agosto de 2015). corponet. Obtenido de http://blog.corponet.com.mx/quees-la-inteligencia-de-negocios

Click2Map (2006-2018). Presentación de la herramienta Click2Map. Recuperado de http://www.click2map.com/

INEC (2016). Anuario de transporte. Recuperado de http://www.ecuadorencifras.gob.ec/documentos/webinec/Estadisticas_Economicas/Estadistica\%20de\%20Transporte/2016/2016_Anuario Transportes_\%20Principales\%20Resultados.pdf

Loor, F. (Enero de 2015). UPWORK. Obtenido de https://www.upwork.com/hiring/development/php-vs-javascript/

Mappinggis (2017). Herramienta Map Marker. Recuperado de https://mappinggis.com/2017/04/opciones-para-crear-mapas-personalizados/

Mozila.org. (Mayo de 2005). mozilla.org. Obtenido de https://developer.mozilla.org/es/docs/Learn/Getting_started_with_the_web/JavaScript _basics

Pérez, D. (7 de 02 de 2015). El Español. Obtenido de https://elandroidelibre.elespanol.com/2015/02/aplicaciones-para-crear-mapaspersonalizados.html

Salazar, J. (Septiembre de 2017). WIKILIBROS.org. Obtenido de https://es.wikibooks.org/wiki/Programaci\%C3\%B3n_en_PHP 